Sobti et al.

\title{
Molecular basis for GIGYF-TNRC6 complex assembly in miRNA-mediated translational repression
}

Meghna Sobti ${ }^{1,2}$, Benjamin J. Mead ${ }^{3}$, Cátia Igreja ${ }^{4}$, Alastair G. Stewart ${ }^{1,2}$, Mary Christie ${ }^{1,2,3,{ }^{*}}$

${ }^{1}$ Molecular, Structural and Computational Biology Division, The Victor Chang Cardiac

Research Institute, Sydney, New South Wales 2010, Australia

${ }^{2}$ St Vincent's Clinical School, Faculty of Medicine, University of New South Wales, Sydney, New South Wales 2052, Australia

${ }^{3}$ School of Life and Environmental Sciences, The University of Sydney, Sydney, New South Wales 2006, Australia

${ }^{4}$ Department of Integrative Evolutionary Biology, Max Planck Institute for Developmental Biology, Max-Planck Ring 9, D-72076 Tübingen, Germany

*Correspondence: tara.christie@sydney.edu.au

Running title: Structure of GIGYF-TNRC6 complexes

Keywords: gene regulation, translational repression, silencing, X-ray crystallography 
Sobti et al.

\begin{abstract}
The GIGYF proteins associate with 4EHP and RNA-associated proteins to elicit transcript-specific translational repression. However, the mechanism by which the GIGYF1/24EHP complex is recruited to its target transcripts remain unclear. Here we report the crystal structures of the GYF domains from GIGYF1 and GIGYF2 in complex with proline-rich sequences from miRISC-binding proteins TNRC6C and TNRC6A, respectively. The TNRC6 proline-rich motifs bind to a conserved array of aromatic residues on the surface of the GIGYF1/2 GYF domain, bridging 4EHP to Argonaute-miRNA mRNA targets. Our structures also reveal a phenylalanine residue conserved from yeast to human GYF domains that contributes to GIGYF2 thermostability. The molecular details we outline here are likely to be conserved between GIGYF1/2 and other RNA-binding proteins to elicit 4EHP-mediated repression in different biological contexts.
\end{abstract}


Sobti et al.

\section{Introduction}

Cap-dependent translation initiation requires the assembly of eIF4F, a heterotrimeric complex comprising the RNA helicase eIF4A, the scaffold protein eIF4G and the cap-binding protein eIF4E to the mRNA $5^{\prime} \mathrm{m}^{7} \mathrm{GpppN}$ cap structure. eIF4G not only bridges eIF4A and eIF4E but also recruits the preinitiation complex to capped mRNA, which in turn commences scanning and identification of the start codon (Sonenberg and Hinnebusch 2009; Jackson et al. 2010). Translation initiation can therefore be regulated by factors that modulate these interactions, such as the eIF4E-binding proteins (4E-BPs) that compete with eIF4G binding to eIF4E (Mader et al. 1995; Marcotrigiano et al. 1999), or proteins that recognise the 5' cap structure which do not associate with eIF4G. One such cap-binding protein is 4EHP (eIF4E homologous protein, also known as eIF4E2), which prevents the assembly of the eIF4F complex on target mRNAs to prevent translation initiation (Rom et al. 1998; Joshi et al. 2004; Hernández et al. 2005). In contrast with eIF4E, 4EHP interacts with GIGYF1 (Grb10interacting GYF protein 1) and its paralog GIGYF2 (Morita et al. 2012; Chapat et al. 2017), the interaction of which closely resembles the eIF4E:eIF4G and eIF4E:4E-BP complexes (Peter et al. 2017). Knockout of 4ehp or Gigyf2 in mice results in perinatal and early postnatal mortality, respectively (Giovannone et al. 2009; Morita et al. 2012). Different neurodegenerative presentations are also associated with the genetic insufficiency or loss of GIGYF1/2 in animals and humans including schizophrenia, autism spectrum disorders and agerelated neurodegeneration (Giovannone et al. 2009; Iossifov et al. 2014; Krumm et al. 2015; Thyme et al. 2019; Satterstrom et al. 2020).

Unlike eIF4G which recruits the translation initiation machinery, human GIGYF proteins have been shown to associate with proteins involved with mRNA degradation (eg. the carbon catabolite repression-negative on TATA-less [CCR4-NOT] deadenylation complex; 
ZFP36/tristetraprolin [TTP]), translational repression (DEAD-box RNA helicase DDX6), mRNA decapping (DDX6, PatL1), ribosome quality control (eg. ZNF598), and miRNAmediated silencing (eg. TNRC6 proteins) (Ash et al. 2010; Morita et al. 2012; Fu et al. 2016; Schopp et al. 2017; Amaya Ramirez et al. 2018; Peter et al. 2019; Ruscica et al. 2019; Tollenaere et al. 2019; Mayya et al. 2021). TNR6CA (also known as GW182), TNR6CB and TNRC6C are Argonaute-binding protein scaffolds that have been implicated in mRNA degradation and translational repression of miRNA targets (Jonas and Izaurralde 2015).

Mutational analysis of zebrafish TNRC6A (DrTNRC6A) indicated that a highly conserved PPGL motif located within the TNRC6A silencing domain contributes to translational inhibition but does not affect deadenylation, the first step of mRNA degradation (Mishima et al. 2012). The PPGL motif is also present in the human TNRC6 proteins, as well as TNRC6/GW182 proteins in other organisms, suggesting that this motif might play a conserved role in TNRC6/GW182-mediated translational repression across metazoa (Mishima et al. 2012). A direct interaction has been observed between TNRC6A and a fragment of GIGYF2 that encompasses its central GYF domain (Schopp et al. 2017). Knockdown of GIGYF2 affects miRNA-mediated translational repression of an mRNA reporter, indicating that GIGYF2 is a regulator of Argonaute-miRNA (miRISC) activity (Schopp et al. 2017).

The human GIGYF proteins were named after their GYF adaptor domains which recognise proline-rich sequences (PRSs) conforming to the PPGФ consensus (where $\Phi$ corresponds to a hydrophobic residue, except for tryptophan) (Kofler et al. 2005; Ash et al. 2010). The GYF domain takes its name from a conserved glycine-tyrosine-phenylalanine $(G Y F)$ motif that is part of the larger GFP-X $\mathrm{X}_{4}-[\mathrm{M} / \mathrm{V} / \mathrm{I}]-\mathrm{X}_{2}-\mathrm{W}-\mathrm{X}_{3}-\mathrm{GYF}$ signature characteristic of the PRS-binding region. This hallmark of GYF domains forms a bulge-helix-bulge structural 
element that generates a hydrophobic ligand binding surface (Freund et al. 1999). GYF domains can be further divided into two subfamilies that are named after the proteins from which they were first identified: the splicing factor CD2BP2 (CD2 antigen cytoplasmic tailbinding protein 2) (Nishizawa et al. 1998), and Saccharomyces cerevisiae (Sc) Smy2 (Kofler et al. 2005) which is thought to be the GIGYF2 homolog in yeast. The two subfamilies are distinguished by the length of the $\beta 1-\beta 2$ loop, as well as by the residue located at position 8 of the GYF domain; a longer $\beta 1-\beta 2$ loop and a Trp at position 8 is characteristic of the CD2BP2 family, while a shorter $\beta 1-\beta 2$ loop and an Asp at position 8 typifies the Smy2 class of GYF domains (Kofler and Freund 2006). Only three GYF-domain containing proteins are encoded in the human genome, namely, CD2BP2, GIGYF1 and GIGYF2.

The specificity of 4EHP-mediated translational repression is thought to be imparted by the GYF domain of the GIGYF proteins, which serves as an adaptor to bridge RNA-binding proteins to 4EHP (Morita et al. 2012; Fu et al. 2016; Schopp et al. 2017; Weber et al. 2020). Mutations that prevent TTP from binding to the GIGYF2 GYF domain impair TTP-mediated repression in mammalian cells (Fu et al. 2016; Peter et al. 2019). Moreover, the repression activity of zebrafish TNRC6A/GW182 can be reduced by mutation of the conserved PPGL motif (Mishima et al. 2012). Mutations within the GIGYF GYF domains that prevent interaction with PPGФ-containing sequences correspondingly disrupt their recruitment to endogenous partners (Weber et al. 2020). The importance of the GIGYF GYF domain is further illustrated in tethering assays whereby the isolated GIGYF2 GYF domain displays repressive activity comparable to that of the full-length protein (Amaya Ramirez et al. 2018).

To understand the mechanism by which PPG $\Phi$ containing proteins are recruited by the GIGYF:4EHP complex, we determined the crystal structures of GIGYF1 and GIGYF2 GYF 
domains in complex with PPGL peptides from TNRC6C and TNRC6A, respectively. These structures highlight the conserved molecular mechanism of PRS recognition by GYF domains and reveal a structural feature in metazoan GYF adaptors that contributes to domain stability.

\section{Results}

PPGФ sequences can directly interact with the isolated GIGYF2 GYF domain.

Recent work has detected a direct interaction between the C-terminal silencing domain of TNRC6C that encodes a PPGФ motif(1369-1690; Fig. 1A) and a central portion of GIGYF2 that encompasses the GYF domain (residues 532-740) (Schopp et al. 2017). The interaction was diminished when the PPGL motif was mutated and when the GYF domain was deleted (residues 607-740 of GIGYF2) (Schopp et al. 2017). We therefore examined whether the GIGYF2 GYF domain (residues 529-597) could alone interact with the isolated TNRC6C PPGL motif (residues 1470-1480, Fig. 1B, blue dashed box). Using glutathione-S-transferase (GST)-tagged TNRC6C PPGL motif, a direct interaction was detected in pull-down assays with His6-tagged GIGYF2 GYF domain (Fig. 1C). Similarly, GST-tagged PPGL fragments of TNRC6A and TNRC6B (Fig. 1B) pulled down the His 6 -GIGYF2 GYF domain (Fig. 1C). This analysis is consistent with interactions detected in other studies (Schopp et al. 2017), and demonstrates that the isolated GIGYF2 GYF domain is sufficient to directly bind to PPGL motifs of the TNRC6 paralogs.

Direct interactions have also been observed between full-length GIGYF2 and TTP from mouse (Fu et al. 2016). We therefore tested whether this interaction was conserved in humans. A GST-tagged fragment of the first tetra-proline region of TTP (residues 68-78, Fig. 1B), which encodes a PPPPGF motif, could also interact with the GIGYF2 GYF domain (Fig. 1C). 
Sobti et al.

PPGФ sequences can directly interact with the isolated GIGYF1 GYF domain.

Co-immunoprecipitation assays using U2OS cells have detected an interaction between full-length GIGYF1 and TTP (Tollenaere et al. 2019). Deletion of the GYF domain or disruption of the characteristic 'GYF' motif in the context of the full-length GIGYF1 protein abolished its interaction with TTP, as well as with ZNF598 which contains three PPPPGF motifs (Tollenaere et al. 2019). Furthermore, a fragment encompassing the GYF domain of GIGYF1 (residues 260-540) was sufficient to bind to full-length ZNF598, and mutation of the three ZNF598 PPPPGF motifs could abrogate this interaction in U2OS cells (Tollenaere et al. 2019). As these results indicate that the GYF domain of GIGYF1 might interact with PPG $\Phi$ sequences in a similar manner to GIGYF2, we tested whether the isolated GIGYF1 GYF domain could also directly interact with the PPGФ motifs from the TNRC6 proteins and TTP.

His6-tagged GIGYF1 GYF domain (residues 470-538; Fig. 1A) interacted with GSTtagged TNRC6 peptides (Fig. 1D). Similar to that observed for GIGYF2, the GST-tagged TTP peptide also directly interacted with the GIGYF1 GYF domain (Fig. 1D).

The GIGYF1/2 GYF domains share structural characteristics typical of the Smy2 subclass of GYF adaptors

While molecular details are available for the CD2BP2 class of GYF domains in humans (Freund et al. 1999; Freund et al. 2002), no structural information is currently available for the human Smy2 class of GYF domains, namely the GYF domains from GIGYF1 and GIGYF2. We therefore determined the crystal structures of the isolated GYF domains from GIGYF2 and GIGYF1 in complex with PPGL peptides from TNRC6A and TNRC6C, respectively (Fig. 2A, 2B; Table S1). Crystals of the TNRC6C-GIGYF1 GYF domain complex were obtained in the C121 space group and diffracted to $1.79 \AA$ resolution. The asymmetric unit comprises two 
TNRC6C-GIGYF1 complexes that display high similarity (rmsd of $0.4 \AA$ over $61 \mathrm{C} \alpha$ atoms). The TNRC6A-GIGYF2 GYF domain complex crystallized in the P $2{ }_{1} 2{ }_{1}{ }_{2}$ space group with one heterodimer in the asymmetric unit, and the structure was refined to $1.23 \AA$ resolution.

The GYF domains of GIGYF1 and GIGYF2 are comprised of a four-stranded antiparallel $\beta$-sheet with two $\alpha$-helices that pack onto one face (Fig. 2A, 2B). The structures of the GYF domains from the human GIGYF proteins are highly conserved (Fig. 2C) with a backbone rmsd of $1.2 \AA$ over $60 \mathrm{C} \alpha$ atoms. The human GIGYF structures also display a high degree of structural similarity with ScSmy2 GYF domain with rmsd values of $1.1 \AA$ and $1.3 \AA$ for GIGYF1 and GIGYF2, respectively (over 56 and $59 \mathrm{C} \alpha$ positions). The secondary structures superimpose well, though there are some deviations in the $\beta 3-\beta 4$ loop, as well as the C-terminus of the domains whereby ScSmy2 contains an additional $\alpha 3$ helix (Fig. 2C).

One of the defining features of the Smy2-subclass of GYF domains is a conserved Asp residue (D540 in GIGYF2, D481 in GIGYF1, and D212 in ScSmy2; denoted by red arrow, Fig. $2 \mathrm{G}$ ) within the $\beta 1-\beta 2$ loop (Kofler and Freund 2006; Ash et al. 2010). In our structures, GIGYF2 D540 and GIGYF1 D481 interact with a hydrogen bond donor present on the $\beta 2$ strand (Q546 in GIGYF2, and Q487 in GIGYF1), which is analogous to H218 in ScSmy2 (Fig. 2D-2F). The orientation of D540 and D481 in the human GIGYF proteins is further stabilised by hydrogen bonds with the backbone amides of the $\beta 1-\beta 2$ loop (Q483 and E485 in GIGYF1, and Q542 and E544 in GIGYF2; Fig. 2D, 2E). Identical interactions are observed for D212 in the $S c$ Smy2 structure (Fig. 2F).

The GIGYF1 and GIGYF2 GYF domains interact with PPGL sequences 
The crystals of human GIGYF GYF domains were obtained in complex with PPGL peptides from TNRC6A and TNRC6C, and the peptides could be built unambiguously into strong density located at the canonical PRS-binding surface (Fig. 2A, 2B, S1A and S1B). A series of residues in the GYF domains form a hydrophobic PRS-binding groove (Fig. 3A, 3B). These residues are Y538, F549, W557, Y562, F563, L567 in GIGYF2, and Y479, F490, W498, Y503, F504, L508 in GIGYF1 (Fig. 3A, 3B). Two hydrogen bonds are formed between GIGYF2 residues Y538 and W557 and the TNRC6A peptide backbone (Fig. 2A). Analogous interactions are observed in the GIGYF1-TNRC6C (Fig. 2B), ScSmy2-BBP and CD2BP2-BD2 complex structures (Freund et al. 2002; Ash et al. 2010).

The TNRC6A PPGФ motif adopts a polyproline II (PPII) helix with P1481 and P1482 interacting with the hydrophobic surface comprised of GIGYF2 Y538, F549, W557 and Y562. In this conformation, P1481 stacks against W557 in an aromatic pocket formed by F549 and Y562 (Fig. 3A). The TNRC6C PPGФ motif similarly forms a PPII helical conformation, with P1475 stacking against W498 of GIGYF1 (Fig. 3B). The requirement for the Gly residue within the PPGФ consensus is structurally rationalized as any larger side chain would clash with the conserved D540 and Q546 of the GIGYF2 GYF domain (or D481 and Q487 in GIGYF1, respectively) (Ash et al. 2010).

The Gly residue within the PPGФ motif facilitates the adoption of turn in trajectory, enabling the subsequent hydrophobic residue $(\Phi)$ to insert into a hydrophobic cavity lined by Y538, F563 and L567 of GIGYF2 (Y479, F504 and L508 in GIGYF1). This cavity is surface exposed owing to the conformation of the characteristic Smy2-subclass Asp residue that orients away from this surface in GIGYF2, GIGYF1 and ScSmy2 (boxed in Fig. 3A-3C). By contrast, the CD2BP2 subfamily of GYF domains contain a Trp in place of the Asp in the $\beta 1-\beta 2$ loop 
(Fig. 2G red arrow; boxed in Fig. 3D), which acts as a cover to obscure the hydrophobic surface formed by Y285, V317 and F313 (Fig. 3D) (Freund et al. 2002; Kofler and Freund 2006; Ash et al. 2010). The different surfaces created by the defining Asp and Trp residues in the Smy2 and CD2BP2 subfamilies, respectively, thereby determines the PRS binding specificities of the GYF domains by either extending or discontinuing the hydrophobic PRS binding surface (Ash et al. 2010).

TNRC6A and TNRC6C both contain Leu residues within their PPGФ motifs, which are inserted into the Smy2 subclass-specific cavity of the GIGYF GYF domains. The TNRC6 peptides bind to the GYF adaptors in a manner highly similar to that observed when $S c \mathrm{BBP}$ interacts with ScSmy2 (Fig. 4C) (Ash et al. 2010). Our structures, together with the ScSmy2ScBBP complex structure (Ash et al. 2010), reveal that the Smy2 subclass-specific cavity can accommodate larger hydrophobic sidechains, such as the Phe observed in the TTP and ZNF598 PPGФ motifs (Fig. 1B). However, the cavity is too small to fit the Trp indole moiety without rearrangements within the binding pocket, consistent with the consensus identified by phage display analyses (Kofler et al. 2005).

A conserved C-terminal Phe side chain is inserted into a hydrophobic pocket in GYF domains from yeast to humans.

The N-terminal half of the GYF domains, comprising $\beta 1, \beta 2, \alpha 1$ and $\alpha 1-\beta 3$ loop structural elements, are responsible for ligand binding and are highly conserved between GYF domain family members (Kofler and Freund 2006). By contrast, the C-terminal regions of GYF domains are poorly conserved, differing in length and composition (Kofler and Freund 2006). This is exemplified by $S c$ Smy2 which contains a C-terminal $\alpha 3$ helix (Ash et al. 2010) not present in the human GIGYF1/2 GYF domains (Fig. 2A-C, 2G). Due to the sequence identity 
Sobti et al.

between the GIGYF1 and GIGYF2 GYF domains ( $80 \%$ identical), even the C-terminal portions are highly similar with only slight differences observed in the conformations $\beta 3-\beta 4$ loop, and the C-terminal loop of the GYF domains (Fig. 4A).

Although there are slight differences in the C-terminal loop between the human GIGYF domain structures, the position of a Phe side chain adopts highly similar positions in GIGYF1 and GIGYF2 (F533 and F592, respectively; Fig. 4A denoted by red asterisk). Superimposition of the human GIGYF structures with $S c$ Smy2 reveals an analogous Phe side chain at this position encoded at the start of the $\alpha 3$ helix (Fig. 4B, red asterisk), despite the conformational variability observed between yeast and human proteins in this region. The structure of the ScSmy2 GYF domain has previously been determined in a domain-swapped arrangement (PDB IDs 3K3V and 3FMA) with C-terminal $\alpha 3$ helices interacting with an adjacent GYF domain. Interestingly, the interactions between the F275 side chain are maintained in the domain-swapped conformation, which is inserted into the hydrophobic pocket of a neighbouring GYF domain (Ash et al. 2010). Moreover, a phenyl moiety is also observed in a similar orientation in the CD2BP2 GYF domain notwithstanding the large differences observed between the C-terminal regions of CD2BP2 and the Smy2-classes of GYF domains (Fig. 4C, red asterisk).

Close inspection of the C-terminal Phe residues in GIGYF1/2, ScSmy2 and CD2BP2 (F592, F533, F275 and F337 in GIGYF2, GIGYF1, ScSmy2 and CD2BP2, respectively) reveals that the Phe side chain is inserted, like a plug, into a hydrophobic cavity that is formed between the $\alpha 1$ and $\alpha 2$ helices and the inside face of the $\beta$-sheet (Fig. 5A-D). In this position, the conserved C-terminal Phe, which we term the 'Phe plug', forms part of the hydrophobic core of the small adaptor domains. Examination of GIGYF and CD2BP2 sequences from yeast 
to humans reveal strict conservation of this C-terminal Phe plug residue (Fig. 2G, and S2 denoted by a red asterisk) in both GIGYF and CD2BP2 GYF domain sequences. While this feature is not annotated as part of the GYF domain in some online databases, the general position of Phe plug was predicted by AlphaFold (Fig. S3A, S3B) (Jumper et al. 2021).

The Phe plug is not conserved in Arabidopsis GYF domains

By contrast, the Phe plug is absent in the only plant GYF domain who structure has been determined (from gene AT5G08430; Fig 5E, PDB code 1WH2). The Arabidopsis thaliana AT5G08430 GYF domain is more divergent from the human GIGYF structures than ScSmy2 and CD2BP2 with larger RMSD values (2.4 $\AA$ and $2.3 \AA$ for GIGYF1 and GIGYF2, respectively) and lower DALI server Z-scores (Z scores above 6.2 for CD2BP2 and ScSmy2, and lower than 5.8 for AT5G08430).

The AT5G08430 GYF domain is located at the extreme C-terminal end of the protein with no additional residues encoded after the $\alpha 2$ helix (Fig. 2G). Compared with the $\alpha 2$ helix of GIGYF GYF domains, the AT5G08430 $\alpha 2$ helix is positioned closer to the $\alpha 1$ helix. In this orientation, hydrophobic side chains L546, V549 and L550 from the AT5G08430 $\alpha 2$ helix pack against the hydrophobic surface formed by W500, L515 and L518 (Fig. 5E-F). Assessment of other GYF or GYF-like domain sequences from A. thaliana identifies a Phe residue directly C-terminal to the $\alpha 2$ helix in three SWIB/PHD/GYF-domain containing proteins (AT2G18090, AT2G16485/NERD, AT3G51120; highlighted by dashed red box in Fig. S3C). However, other A. thaliana GYF domain-containing proteins (AT5G42950/EXA1, AT1G24300 and AT1G27430), for which no structures have yet been determined, do not encode obvious Phe plug residues at the C-terminus of their GYF domains. Notably, the GIGYF ortholog in A. thaliana, AT5G42950/EXA1, encodes a PPPGF sequence in this region 
Sobti et al.

that is thought to act as an autoinhibitory sequence to prevent low affinity interactions with the GYF domain (boxed blue in Fig. S3C) (Kofler and Freund 2006).

\section{The Phe plug contributes to GIGYF2 GYF domain stability.}

To investigate the importance of the conserved C-terminal Phe plug, we generated GYF domain mutants that disrupted the hydrophobic nature of the plug. More specifically, we replaced the Phe plug with the negatively-charged Glu residue generating GIGYF1 F592E and GIGYF2 F533E mutants. The His 6 -tagged mutant GYF domains were then tested for their ability to bind to the PRS sequences of TNRC6A and TTP. As the Phe residue is distal to the PRS binding site, the substitution did not affect the binding of PRS-containing sequences in GST pull-down assays under the conditions tested (Fig. S3D, S3E). Thermal stability assays, however, indicated that mutation of the Phe plug decreased the stability of the isolated GIGYF2 GYF domain with the apparent melting temperature of the F533E mutant 7 degrees lower than that observed for the wildtype domain $\left(\mathrm{Tm}_{\mathrm{app}}\right.$ of $61^{\circ} \mathrm{C}$ and $54{ }^{\circ} \mathrm{C}$ for the wildtype and F533E GYF domains, respectively; Fig. S4).

\section{Discussion}

In this study, we have elucidated the molecular basis of TNRC6 recognition by human GIGYF1/2 GYF domains, an interaction that enables 4EHP-mediated translational repression of miRNA-bound mRNA transcripts. While this study focuses on the TNRC6 proteins and TTP, other PRS-containing factors involved in translational regulation are known to interact with the GIGYF proteins in eukaryotic cells (Ash et al. 2010; Morita et al. 2012; Amaya Ramirez et al. 2018; Ruscica et al. 2019; Tollenaere et al. 2019; Mayya et al. 2021). As such, the molecular details we outline here are likely to be conserved in a variety of biological contexts to elicit 4EHP-mediated repression. 
Sobti et al.

Using pull-down assays, we have demonstrated that the isolated GYF domains of GIGYF1 and GIGYF2 directly interact with PPGФ-containing sequences from binding partners involved in post-transcriptional gene expression regulation, and that these interactions are conserved between the human GIGYF paralogs. This work extends previous studies by refining the minimal molecular requirements of the GIGYF-TNRC6/TTP interactions. Our structures reveal that the overall features of the Smy2 subfamily of GYF domains are conserved between yeast and humans, and rationalise how PPGF motifs, which are present in TTP and ZNF598, can bind to the GIGYF1/2 GYF domain surface. Comparison of our structures with reported GYF domains reveal the presence a conserved Phe plug at the C-terminus CD2BP2 and Smy2 subclasses of GYF domains from yeast to humans. Sequence analyses, however, indicate that this feature may not be present in A. thaliana GYF domains. Mutational analyses of the Phe plug in GIGYF1/2 suggests that this feature contributes to the stability of the GIGYF2 GYF domain, but the integrity of the Phe plug residue is not strictly required for PRS binding in vitro. This is consistent with the observation that the Phe plug is not present in the A. thaliana AT5G08430 GYF, although it is currently unclear if this domain can bind to PRScontaining proteins in plants. These results therefore refine our understanding of the molecular features of GYF domain adaptors, which should be taken into consideration when designing GYF domain constructs.

The "RPPPGL" sequence shared between TNRC6A and TNRC6C adopt similar conformations in our structures (Fig. 2A, 2B). It is therefore highly likely that TNRC6A interacts with GIGYF1 in a similar manner to that observed for TNRC6C, and, likewise, that TNRC6C interacts in an analogous manner to TNRC6A when binding to GIGYF2, corroborated by the similar binding properties seen in our pulldown studies (Fig. 1C, 1D). The 
"RPPPGL" motif is also present in TNRC6B (Figure 1B) (Mishima et al. 2012), and given the high similarity between the GIGYF2-TNRC6A and GIGYF1-TNRC6C structures, we would expect that comparable interactions would be mediated between TNRC6B and the GIGYF1/2 GYF domains. More broadly, the residues that comprise the GIGYF PRS-binding site, and the TNRC6/GW182 "PPGL” motif, are highly conserved from cnidaria (Nematostella vectensis; $N v$ ) to humans (Fig. 1B, 2G). This indicates that the structures determined here would be suitable models of GIGYF-GW182 interactions in different species.

Mutations in GIGYF2 have been implicated in neurological conditions such as autism spectrum disorder, schizophrenia, and Parkinson's disease (Lautier et al. 2008; Iossifov et al. 2014; Krumm et al. 2015; Thyme et al. 2019; Satterstrom et al. 2020). Although the association of GIGYF2 mutants in Parkinson's disease remains controversial (Bras et al. 2009; Nichols et al. 2009; Tan and Schapira 2010), recent work has revealed the importance of the GIGYF24EHP axis in protein quality control (Hickey et al. 2020; Juszkiewicz et al. 2020; Sinha et al. 2020; Weber et al. 2020). It is therefore tempting to speculate that the impairment of GIGYF2 function may lead to the accumulation of misfolded and potentially cytotoxic polypeptide products that contribute to the development of neurological conditions. L580F is a GIGYF2 GYF domain mutation identified in Parkinson's disease patient cohorts (Wang et al. 2010). As L580 forms part of the Phe plug binding pocket, the L580F mutation may therefore affect the packing of the GYF domain hydrophobic core. It will now be interesting to determine what effect the L580F mutation has on GYF domain stability and PRS-binding capacity.

\section{Materials and Methods}

Protein expression and purification 
GST-PPGL and GST-TTP fragments were expressed in BL21(DE3) cells overnight at $18^{\circ} \mathrm{C}$. The GST-fusion proteins were purified in $50 \mathrm{mM}$ Tris $7.5,125 \mathrm{mM} \mathrm{NaCl}, 2 \mathrm{mM} 2-$ mercaptoethanol (BME). His6-GIGYF2 GYF domain was expressed in BL21(DE3) cells at $20^{\circ} \mathrm{C}$ overnight, and pellets were resuspended in $50 \mathrm{mM}$ Tris $8.0,400 \mathrm{mM} \mathrm{NaCl}, 2 \mathrm{mM}$ imidazole, 5 mM BME. The His ${ }_{6}$-GIGYF2 GYF domain was eluted from a Ni-NTA column (GE Healthcare) using $250 \mathrm{mM}$ imidazole and further purified using a S200 10/600 column (GE Healthcare) and flash frozen in $10 \mathrm{mM}$ Tris 8.0, $150 \mathrm{mM} \mathrm{NaCl}, 5 \mathrm{mM} \mathrm{BME.} \mathrm{His} 6$-GIGYF1 GYF domain was expressed and purified in similar conditions to GIGYF2. His6-GIGYF1/2 Phe plug mutants (F592E and F533E, respectively) were expressed and purified using similar conditions as the wildtype domains. Both wildtype and F592E mutant GIGYF1 GYF domains appeared to be less stable than the isolated GIGYF2 GYF domains. GIGYF2-TNRC6A complexes were obtained by immobilising GST-TNRC6A PPGL fragments on a GSTrap column and binding to purified GIGYF2 protein. The complex was eluted using $10 \mathrm{mM}$ glutathione, and the GST tag was cleaved overnight using GST-3C protease. The complex was purified from GST using size exclusion chromatography. GIGYF1-TNRC6C complexes were purified using similar conditions.

\section{Crystallisation and data collection}

Crystals of GIGYF2-TNRC6A were obtained in 1.0-1.4 M Na/K phosphate $\mathrm{pH}$ 7.4-7.8 and were flash frozen in liquid nitrogen using $25 \%$ glycerol as a cryoprotectant. Crystals of GIGYF1-TNRC6C were obtained in $100 \mathrm{mM}$ Hepes 7.0, $1 \mathrm{M}$ sodium malonate, and 25\% glycerol was used as a cryoprotectant before freezing. Data were collected at the Australian Synchrotron MX2 beamline (McPhillips et al. 2002; Aragão et al. 2018) and processed using XDS (Kabsch 2010). For the GIGYF2-TNRC6A complex, ScSmy2 was used as a molecular replacement model with $S c$ BBP coordinates removed (PDB ID 3FMA) (Ash et al. 2010). For 
the GIGYF1-TNRC6C, the GIGYF2 structure was used as the search model (TNRC6A coordinates removed) in PHASER (McCoy et al. 2007). Refinement was performed in Phenix (Liebschner et al. 2019), and both structures have excellent geometry (Table S1) (Williams et al. 2018).

GST pull-down

Pull-down assays were performed essentially as described previously. Specifically, 80 $\mu \mathrm{g}$ of GST or GST-PPGL/GST-TTP fragments were incubated with $160 \mu \mathrm{g}$ of His 6 -GIGYF2 or His 6 -GIGYF1 GYF domains in the presence of $25 \mu \mathrm{L}$ of glutathione superflow agarose (Pierce) pre-equilibrated in binding buffer (50 mM Tris 7.5, $125 \mathrm{mM} \mathrm{NaCl}, 2 \mathrm{mM}$ BME). The resin was washed 4 times in binding buffer, and bound proteins were eluted with binding buffer supplemented with $20 \mathrm{mM}$ glutathione. The samples were analysed using SDS-PAGE, input lanes correspond to $2 \%$ of incubated protein and pull-down lanes correspond to $10 \%$ of eluted sample.

\section{Thermal shift assays}

Purified His ${ }_{6}$-GIGYF2 GYF domain, wildtype and F533E mutant in $10 \mathrm{mM}$ Tris 7.5, $150 \mathrm{mM} \mathrm{NaCl}, 5 \mathrm{mM}$ BME were mixed with SYPRO orange (Invitrogen; 10x final concentration) and dispensed into a 96-well qPCR plate. The solution was slowly heated from $20{ }^{\circ} \mathrm{C}$ to $95{ }^{\circ} \mathrm{C}$ using an Applied Biosystems real-time qPCR machine. Fluorescence was detected using a ROX filter. The data were analysed using the 7500 Software (ABI) and the minimum of the negative first derivative was used to determine the apparent melting temperature $\left(\mathrm{Tm}_{\mathrm{app}}\right)$. Assays were performed in triplicate. Similar calculations could not be performed with the isolated wildtype GIGYF1 GYF domain and the F592E mutant which both displayed high initial fluorescence and ambiguous melt transition, consistent with the 
Sobti et al.

instability of the domains observed during purification. Buffer alone and lysozyme was used as negative and positive controls, respectively.

\section{Data deposition}

The coordinates of GIGYF1-TNRC6C (7RUQ) and GIGYF2-TNRC6A (7RUP) have been deposited into the Protein Data Bank.

\section{Acknowledgments}

We thank the beamline scientists at the Australian Synchrotron for their assistance with data collection. This research was undertaken in part using the MX2 beamline at the Australian Synchrotron, part of ANSTO, and made use of the Australian Cancer Research Foundation (ACRF) detector. A.G.S. was supported by a National Health and Medical Research Council Fellowship APP1159347 and Grant APP1146403. M.C. was supported by Australian Research Council Fellowship DE160100608. 
Sobti et al.

\section{References}

Amaya Ramirez CC, Hubbe P, Mandel N, Béthune J. 2018. 4EHP-independent repression of endogenous mRNAs by the RNA-binding protein GIGYF2. Nucleic Acids Research 46: $5792-5808$.

Aragão D, Aishima J, Cherukuvada H, Clarken R, Clift M, Cowieson NP, Ericsson DJ, Gee CL, Macedo S, Mudie N et al. 2018. MX2: a high-flux undulator microfocus beamline serving both the chemical and macromolecular crystallography communities at the Australian Synchrotron. J Synchrotron Radiat 25: 885-891.

Ash M-R, Faelber K, Kosslick D, Albert GI, Roske Y, Kofler M, Schuemann M, Krause E, Freund C. 2010. Conserved $\beta$-Hairpin Recognition by the GYF Domains of Smy2 and GIGYF2 in mRNA Surveillance and Vesicular Transport Complexes. Structure 18: 944-954.

Bras J, Simón-Sánchez J, Federoff M, Morgadinho A, Januario C, Ribeiro M, Cunha L, Oliveira C, Singleton AB. 2009. Lack of replication of association between GIGYF2 variants and Parkinson disease. Hum Mol Genet 18: 341-346.

Chapat C, Jafarnejad SM, Matta-Camacho E, Hesketh GG, Gelbart IA, Attig J, Gkogkas CG, Alain T, Stern-Ginossar N, Fabian MR et al. 2017. Cap-binding protein 4EHP effects translation silencing by microRNAs. Proceedings of the National Academy of Sciences 114: 5425 .

Freund C, Dötsch V, Nishizawa K, Reinherz EL, Wagner G. 1999. The GYF domain is a novel structural fold that is involved in lymphoid signaling through proline-rich sequences. Nature Structural Biology 6: 656-660.

Freund C, Kühne R, Yang H, Park S, Reinherz EL, Wagner G. 2002. Dynamic interaction of CD2 with the GYF and the SH3 domain of compartmentalized effector molecules. The EMBO Journal 21: 5985-5995. 
Fu R, Olsen MT, Webb K, Bennett EJ, Lykke-Andersen J. 2016. Recruitment of the 4EHPGYF2 cap-binding complex to tetraproline motifs of tristetraprolin promotes repression and degradation of mRNAs with AU-rich elements.

Giovannone B, Tsiaras WG, de la Monte S, Klysik J, Lautier C, Karashchuk G, Goldwurm S, Smith RJ. 2009. GIGYF2 gene disruption in mice results in neurodegeneration and altered insulin-like growth factor signaling. Hum Mol Genet 18: 4629-4639.

Hernández G, Altmann M, Sierra JM, Urlaub H, Diez del Corral R, Schwartz P, Rivera-Pomar R. 2005. Functional analysis of seven genes encoding eight translation initiation factor 4E (eIF4E) isoforms in Drosophila. Mechanisms of Development 122: 529-543.

Hickey KL, Dickson K, Cogan JZ, Replogle JM, Schoof M, D’Orazio KN, Sinha NK, Hussmann JA, Jost M, Frost A et al. 2020. GIGYF2 and 4EHP Inhibit Translation Initiation of Defective Messenger RNAs to Assist Ribosome-Associated Quality Control. Mol Cell 79: 950-962.e956.

Iossifov I, O’Roak BJ, Sanders SJ, Ronemus M, Krumm N, Levy D, Stessman HA, Witherspoon KT, Vives L, Patterson KE et al. 2014. The contribution of de novo coding mutations to autism spectrum disorder. Nature 515: 216-221.

Jackson RJ, Hellen CUT, Pestova TV. 2010. The mechanism of eukaryotic translation initiation and principles of its regulation. Nature Reviews Molecular Cell Biology 11: 113-127.

Jonas S, Izaurralde E. 2015. Towards a molecular understanding of microRNA-mediated gene silencing. Nature Reviews Genetics 16: 421-433.

Joshi B, Cameron A, Jagus R. 2004. Characterization of mammalian eIF4E-family members. European Journal of Biochemistry 271: 2189-2203.

Jumper J, Evans R, Pritzel A, Green T, Figurnov M, Ronneberger O, Tunyasuvunakool K, Bates R, Žídek A, Potapenko A et al. 2021. Highly accurate protein structure prediction with AlphaFold. Nature. 
Juszkiewicz S, Slodkowicz G, Lin Z, Freire-Pritchett P, Peak-Chew S-Y, Hegde RS. 2020. Ribosome collisions trigger cis-acting feedback inhibition of translation initiation. Elife 9: e60038.

Kabsch W. 2010. XDS. Acta Crystallogr D Biol Crystallogr 66: 125-132.

Kofler M, Motzny K, Freund C. 2005. GYF Domain Proteomics Reveals Interaction Sites in Known and Novel Target Proteins*S. Molecular \& Cellular Proteomics 4: 1797-1811. Kofler MM, Freund C. 2006. The GYF domain. The FEBS Journal 273: 245-256.

Krumm N, Turner TN, Baker C, Vives L, Mohajeri K, Witherspoon K, Raja A, Coe BP, Stessman HA, He Z-X et al. 2015. Excess of rare, inherited truncating mutations in autism. Nature Genetics 47: 582-588.

Lautier C, Goldwurm S, Dürr A, Giovannone B, Tsiaras WG, Pezzoli G, Brice A, Smith RJ. 2008. Mutations in the GIGYF2 (TNRC15) gene at the PARK11 locus in familial Parkinson disease. Am J Hum Genet 82: 822-833.

Liebschner D, Afonine PV, Baker ML, Bunkoczi G, Chen VB, Croll TI, Hintze B, Hung L-W, Jain S, McCoy AJ et al. 2019. Macromolecular structure determination using X-rays, neutrons and electrons: recent developments in Phenix. Acta Crystallographica Section D 75: 861-877.

Mader S, Lee H, Pause A, Sonenberg N. 1995. The translation initiation factor eIF-4E binds to a common motif shared by the translation factor eIF-4 gamma and the translational repressors 4E-binding proteins. Mol Cell Biol 15: 4990-4997.

Marcotrigiano J, Gingras AC, Sonenberg N, Burley SK. 1999. Cap-dependent translation initiation in eukaryotes is regulated by a molecular mimic of eIF4G. Mol Cell 3: 707716. 
Mayya VK, Flamand MN, Lambert AM, Jafarnejad SM, Wohlschlegel JA, Sonenberg N, Duchaine TF. 2021. microRNA-mediated translation repression through GYF-1 and IFE-4 in C. elegans development. Nucleic Acids Research 49: 4803-4815.

McCoy AJ, Grosse-Kunstleve RW, Adams PD, Winn MD, Storoni LC, Read RJ. 2007. Phaser crystallographic software. Journal of Applied Crystallography 40: 658-674.

McPhillips TM, McPhillips SE, Chiu H-J, Cohen AE, Deacon AM, Ellis PJ, Garman E, Gonzalez A, Sauter NK, Phizackerley RP et al. 2002. Blu-Ice and the Distributed Control System: software for data acquisition and instrument control at macromolecular crystallography beamlines. J Synchrotron Radiat 9: 401-406.

Mishima Y, Fukao A, Kishimoto T, Sakamoto H, Fujiwara T, Inoue K. 2012. Translational inhibition by deadenylation-independent mechanisms is central to microRNA-mediated silencing in zebrafish. Proceedings of the National Academy of Sciences 109: 1104.

Morita M, Ler LW, Fabian MR, Siddiqui N, Mullin M, Henderson VC, Alain T, Fonseca BD, Karashchuk G, Bennett CF et al. 2012. A novel 4EHP-GIGYF2 translational repressor complex is essential for mammalian development. Mol Cell Biol 32: 3585-3593.

Nichols WC, Kissell DK, Pankratz N, Pauciulo MW, Elsaesser VE, Clark KA, Halter CA, Rudolph A, Wojcieszek J, Pfeiffer RF et al. 2009. Variation in GIGYF2 is not associated with Parkinson disease. Neurology 72: 1886.

Nishizawa K, Freund C, Li J, Wagner G, Reinherz EL. 1998. Identification of a proline-binding motif regulating CD2-triggered T lymphocyte activation. Proceedings of the National Academy of Sciences 95: 14897.

Peter D, Ruscica V, Bawankar P, Weber R, Helms S, Valkov E, Igreja C, Izaurralde E. 2019. Molecular basis for GIGYF-Me31B complex assembly in 4EHP-mediated translational repression. Genes Dev 33: 1355-1360. 
Peter D, Weber R, Sandmeir F, Wohlbold L, Helms S, Bawankar P, Valkov E, Igreja C, Izaurralde E. 2017. GIGYF1/2 proteins use auxiliary sequences to selectively bind to 4EHP and repress target mRNA expression. Genes Dev 31: 1147-1161.

Rom E, Kim HC, Gingras A-C, Marcotrigiano J, Favre D, Olsen H, Burley SK, Sonenberg N. 1998. Cloning and Characterization of 4EHP, a Novel Mammalian eIF4E-related Capbinding Protein *. Journal of Biological Chemistry 273: 13104-13109.

Ruscica V, Bawankar P, Peter D, Helms S, Igreja C, Izaurralde E. 2019. Direct role for the Drosophila GIGYF protein in 4EHP-mediated mRNA repression. Nucleic Acids Research 47: 7035-7048.

Satterstrom FK, Kosmicki JA, Wang J, Breen MS, De Rubeis S, An J-Y, Peng M, Collins R, Grove J, Klei L et al. 2020. Large-Scale Exome Sequencing Study Implicates Both Developmental and Functional Changes in the Neurobiology of Autism. Cell 180: 568584.e523.

Schopp IM, Amaya Ramirez CC, Debeljak J, Kreibich E, Skribbe M, Wild K, Béthune J. 2017. Split-BioID a conditional proteomics approach to monitor the composition of spatiotemporally defined protein complexes. Nature Communications 8: 15690.

Sinha NK, Ordureau A, Best K, Saba JA, Zinshteyn B, Sundaramoorthy E, Fulzele A, Garshott DM, Denk T, Thoms M et al. 2020. EDF1 coordinates cellular responses to ribosome collisions. Elife 9: e58828.

Sonenberg N, Hinnebusch AG. 2009. Regulation of Translation Initiation in Eukaryotes: Mechanisms and Biological Targets. Cell 136: 731-745.

Tan EK, Schapira AH. 2010. Summary of GIGYF2 studies in Parkinson's disease: the burden of proof. European Journal of Neurology 17: 175-176. 
Sobti et al.

Thyme SB, Pieper LM, Li EH, Pandey S, Wang Y, Morris NS, Sha C, Choi JW, Herrera KJ, Soucy ER et al. 2019. Phenotypic Landscape of Schizophrenia-Associated Genes Defines Candidates and Their Shared Functions. Cell 177: 478-491.e420.

Tollenaere MAX, Tiedje C, Rasmussen S, Nielsen JC, Vind AC, Blasius M, Batth TS, Mailand N, Olsen JV, Gaestel M et al. 2019. GIGYF1/2-Driven Cooperation between ZNF598 and TTP in Posttranscriptional Regulation of Inflammatory Signaling. Cell Reports 26: 3511-3521.e3514.

Wang L, Guo J-f, Zhang W-w, Xu Q, Zuo X, Shi C-h, Luo L-z, Liu J, Hu L, Hu Y-c et al. 2010. Novel GIGYF2 gene variants in patients with Parkinson's disease in Chinese population. Neuroscience Letters 473: 131-135.

Weber R, Chung M-Y, Keskeny C, Zinnall U, Landthaler M, Valkov E, Izaurralde E, Igreja C. 2020. 4EHP and GIGYF1/2 Mediate Translation-Coupled Messenger RNA Decay. Cell Reports 33: 108262.

Williams CJ, Headd JJ, Moriarty NW, Prisant MG, Videau LL, Deis LN, Verma V, Keedy DA, Hintze BJ, Chen VB et al. 2018. MolProbity: More and better reference data for improved all-atom structure validation. Protein Science 27: 293-315. 
Sobti et al.
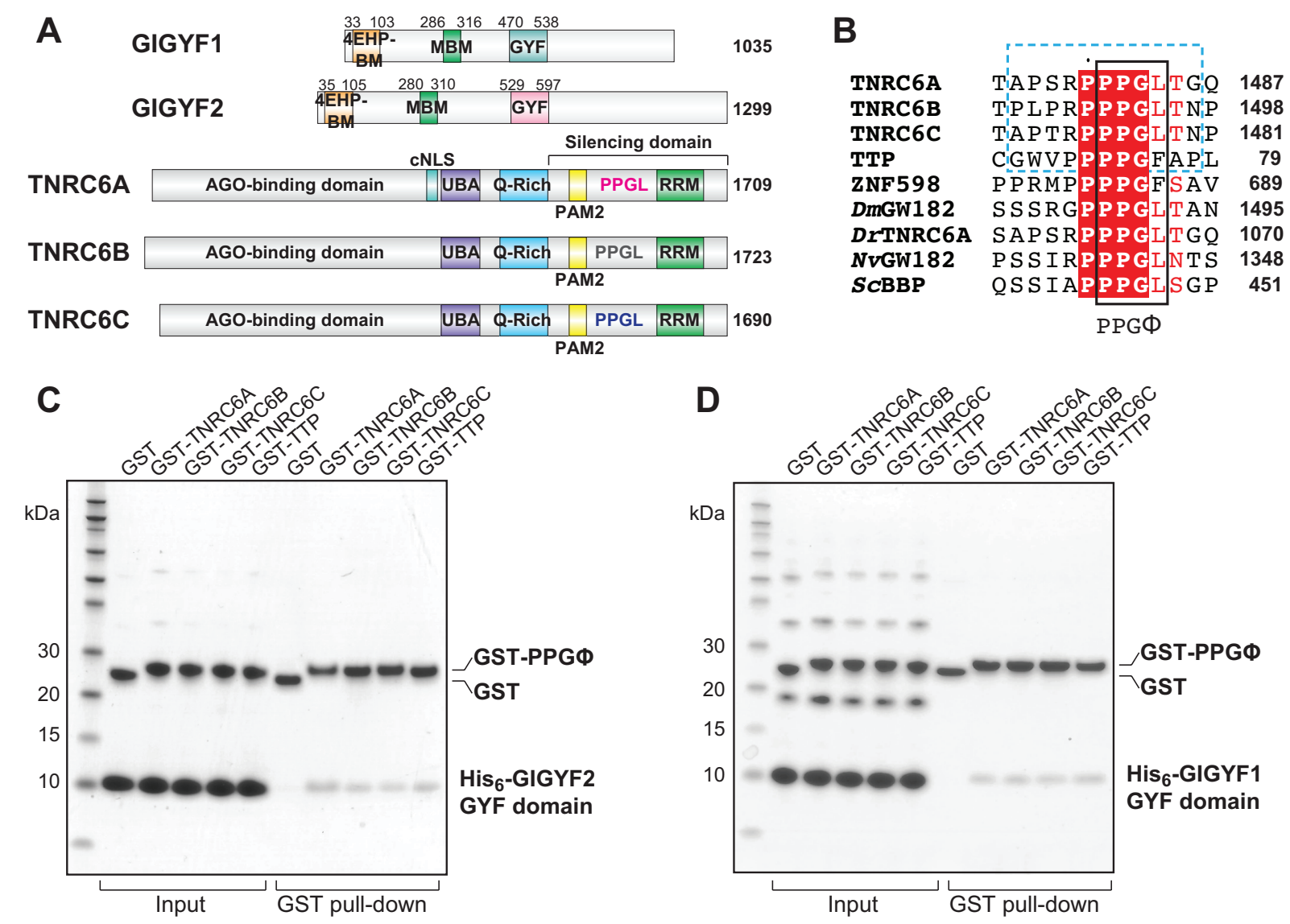

Figure 1. GIGYF1/2 GYF domains directly interact with proline-rich sequences from GW182/TNRC6 proteins. (A) Schematic representation of human GIGYF1/2 and TNRC6A-C proteins. GIGYF1/2 contain a 4EHP-binding motif (4EHP-BM) and a Me31B/DDX6-binding motif (MBM) within their N-terminal region, as well as a central GYF domain. The conserved PPGL motifs of the TNRC6 proteins are located within their C-terminal silencing domains, which also contains a PAM motif and an RNA-recognition motif (RRM) domain. TNRC6A contains a classical nuclear localisation signal (cNLS) between its Argonaute (AGO)-binding domain and ubiquitin-associated motif (UBA). The TNRC6 paralogs also contain Q-rich regions. (B) The PPGL motif is conserved in GW182/TNRC6A proteins in metazoa, and proline-rich sequences have been identified in RNA-binding proteins such as TTP and ZNF598. The PPG $\Phi$ is denoted by the black box, and the 11-residue peptides used in this study are indicated by the blue dashed box. For comparison, the PRS from $S c$ BBP is also shown. The 
bioRxiv preprint doi: https://doi.org/10.1101/2021.08.20.457040; this version posted August 20, 2021. The copyright holder for this preprint (which was not certified by peer review) is the author/funder. All rights reserved. No reuse allowed without permission.

Sobti et al.

species abbreviations are as follows: Dm (Drosophila melanogaster), Dr (Danio rerio), $N v$ (Nematostella vectensis), Sc (Saccharomyces cerevisiae). (C and D) GST pull-downs using recombinant His6-GIGYF1/2 GYF domain and GST-PRS sequences. GST only served as a negative control. 

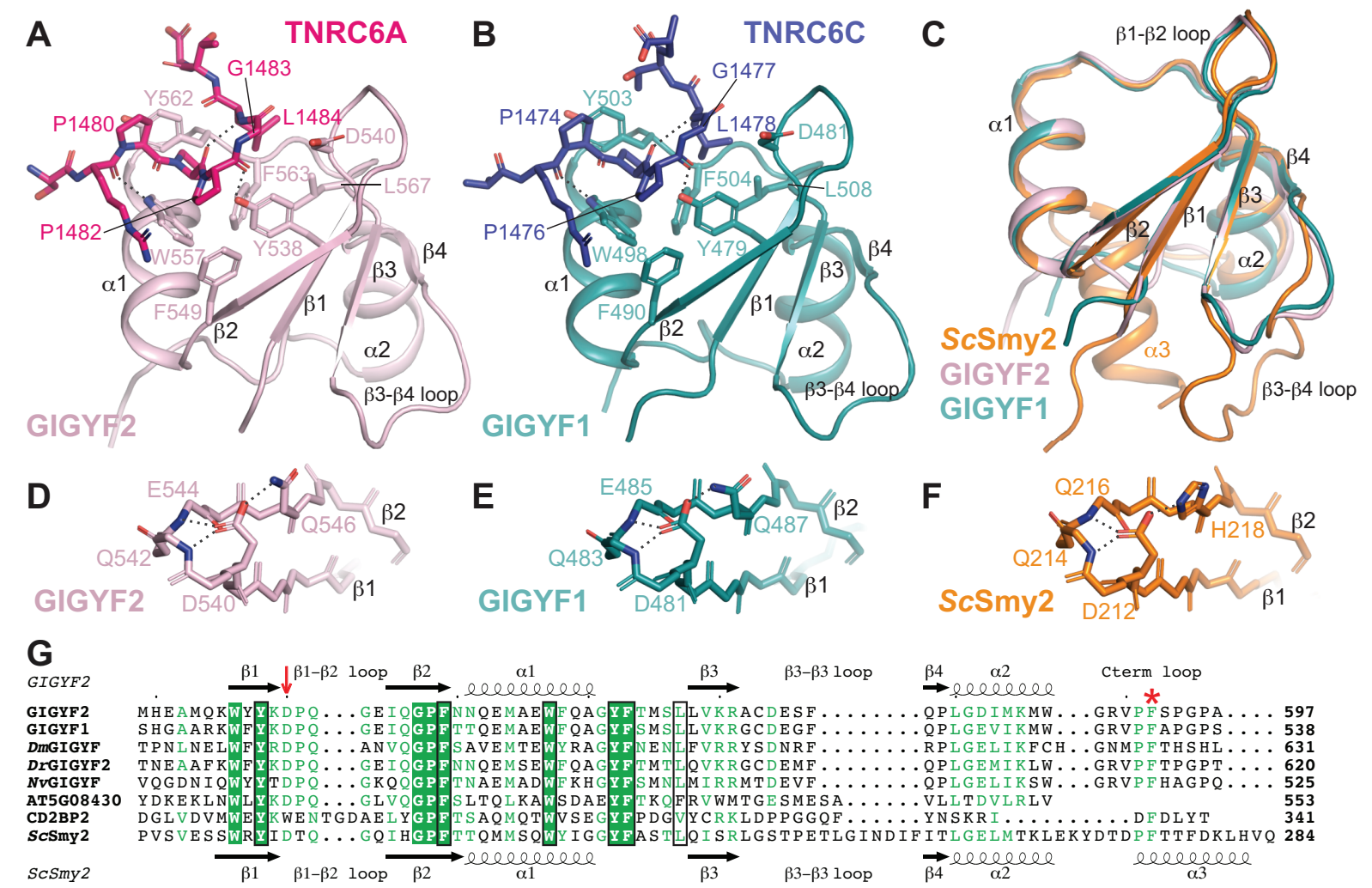

Figure 2. Overall structures of GIGYF1/2 GYF domains in complex with TNRC6 PPGLcontaining peptides. (A) The TNRC6A PRS peptide (hot pink sticks) binds to a conserved arrange of aromatic residues on the surface of the GIGYF2 GYF domain (light pink cartoon). Hydrogen bonds are denoted by dashed lines. Similar interactions are observed between the TNRC6C PRS peptide (navy sticks) and the GIGYF1 GYF domain (teal cartoon) (B). (C) The GIGYF1/2 GYF domains are highly similar to the ScSmy2 GYF fold (PDB ID 3FMA). (D-F) The conserved Asp residue that defines the Smy2 class of GYF domains mediates analogous interactions in GIGYF1/2 and ScSmy2. (G) Structural-based sequence alignment of GYF domains. The prototypical Asp residue of the Smy2 GYFs is indicated by the red arrow. The residues that comprise the PRS-binding surface are boxed in black. The Phe plug is denoted by the red asterisk. 
Sobti et al.
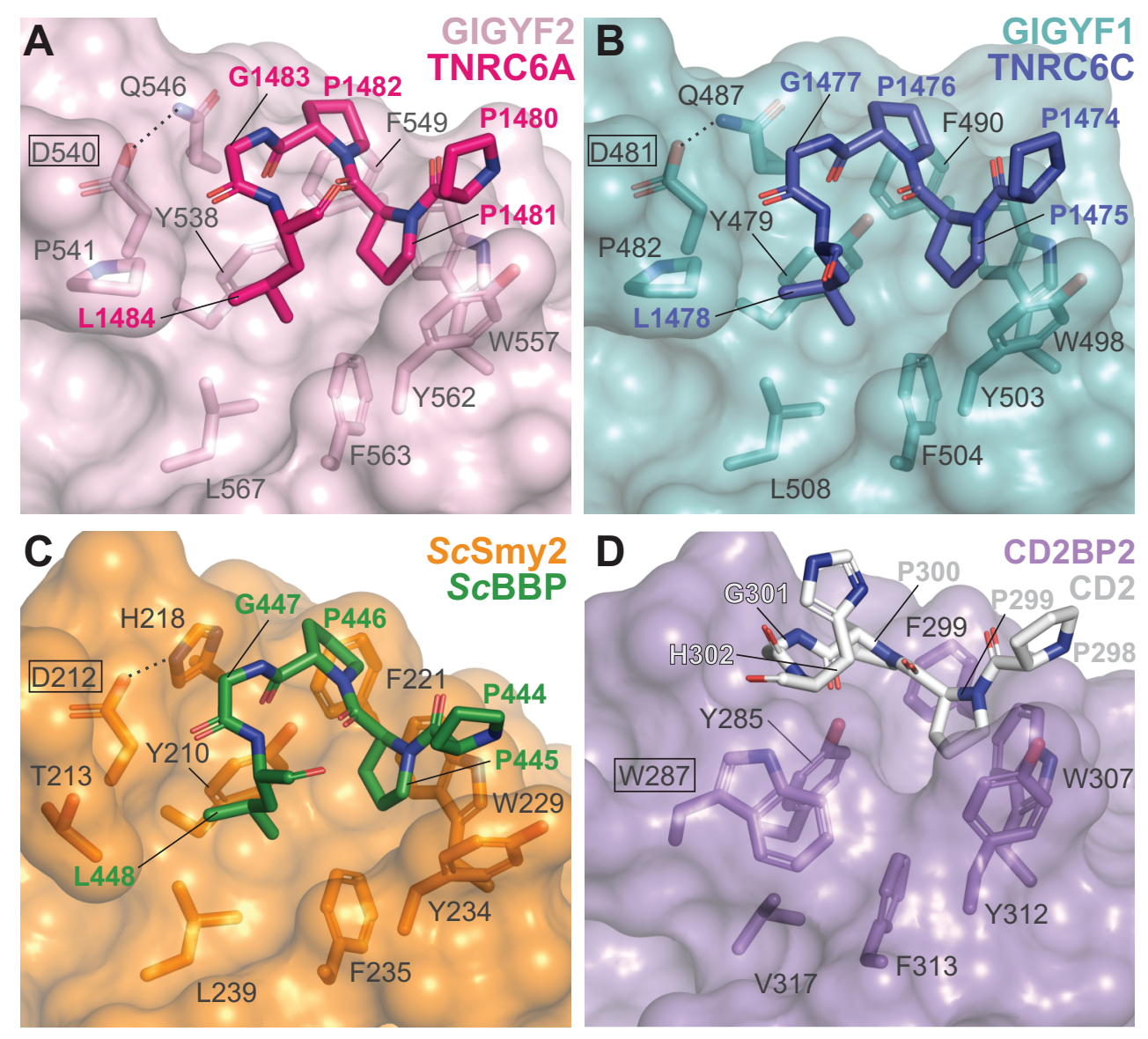

Figure 3. Proline-rich sequences bind to a conserved hydrophobic depression of the GYF domain surface. (A-C) Close-up views of TNRC6A, TNRC6C and ScBBP peptides binding to the GYF domains of GIGYF2, GIGYF1 and ScSmy2 (PDB ID 3FMA), respectively. The defining Asp residue of the Smy2 class of GYF domains are indicated by black boxes. By contrast, the CD2BP2 class of GYF domains contain a Trp (W287 in human CD2BP2, boxed; PDB ID 1L2Z) at this position (D). The conserved Trp in CD2BP2 GYF domains disrupt the hydrophobic cavity, which is exploited by PRS-containing sequences in the Smy2 class of GYF domains. 
Sobti et al.
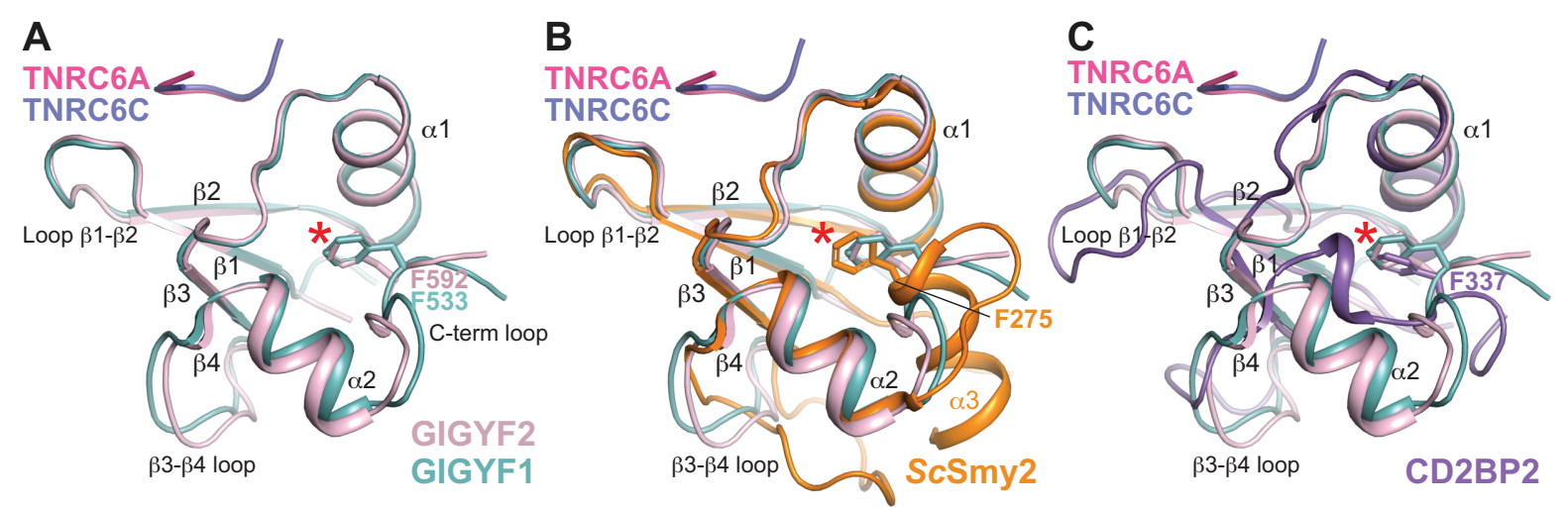

Figure 4. A conserved Phe residue is found at the C-terminus of Smy2 and CD2BP2 GYF domain classes from yeast to humans. (A) Superimposition of GYF domains from GIGYF1 and GIGYF2, shown in teal and light pink, respectively. The TNRC6 peptides are shown in hot pink and navy cartoon representation, The conserved Phe residue is shown in sticks and indicated by red asterisk. The same view is shown in (B) and (C) but with ScSmy2 (orange; PDB ID 3FMA) and CD2BP2 (purple; PDB ID 1L2Z) superimposed, respectively. 


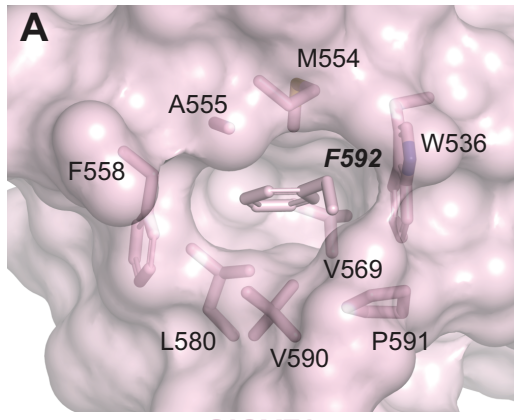

GIGYF2

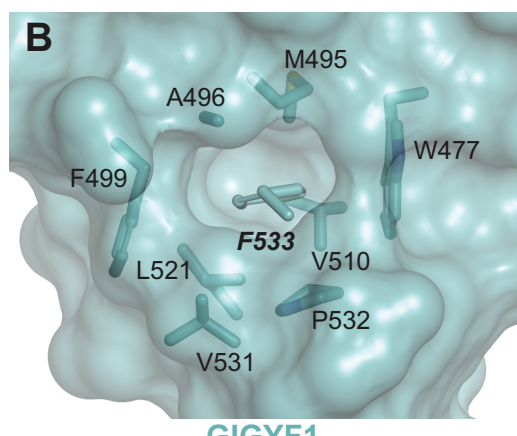

GIGYF1

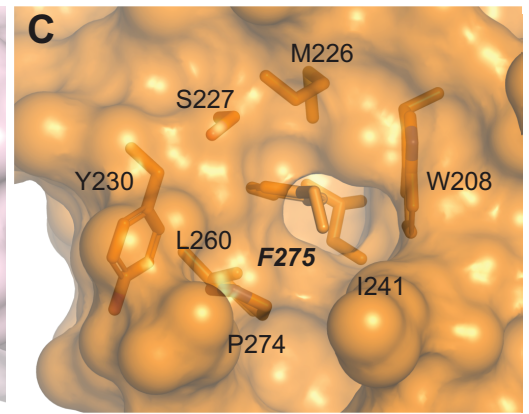

ScSmy2

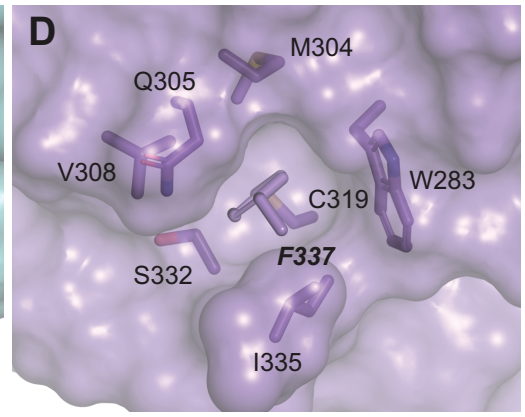

CD2BP2

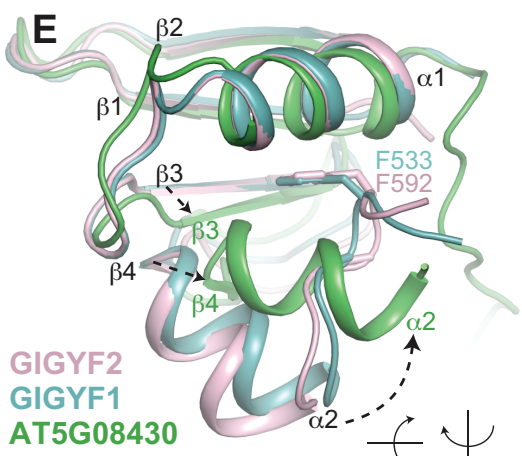

AT5G08430

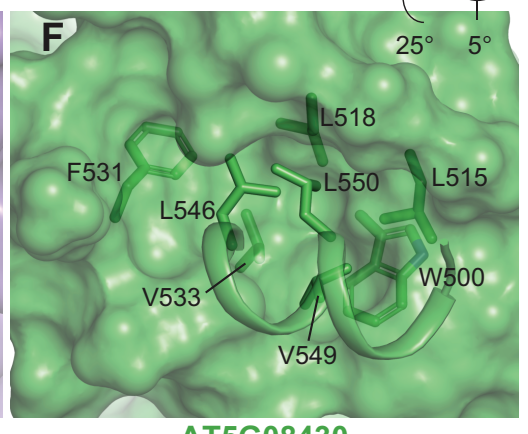

AT5G08430

Figure 5. The conserved Phe plug forms part of a hydrophobic core in Smy2 and CD2BP2 classes of GYF domains from yeast to humans. (A to D) Surface representation of GIGYF2, GIGYF1, ScSmy2 (PDB ID 3FMA) and CD2BP2 (PDB ID 1L2Z), respectively, with residues surround the Phe plug shown in sticks. (E) Superimposition of GIGYF2 and GIGYF1 (light pink and teal cartoon, respectively) with A. thaliana ATG08430 (green; PDB ID 1WH2). The GYF domain of A. thaliana ATG08430 is found at the extreme C-terminal end of the protein and does not encode a Phe plug residue. Conformational changes are observed between the $A$. thaliana ATG08430 and GIGYF1/2 in the $\alpha 2$ helix, as well as the $\beta 3$ and $\beta 4$ strands of the GYF domain. (F) The $\alpha 2$ helix in the plant GYF domain packs against the hydrophobic surface in the absence of a Phe plug. 